\title{
Effect of Quantum Gravity on the Stability of Black Holes
}

\author{
Riasat Ali ${ }^{1}$, Kazuharu Bamba ${ }^{2, *}$ and Syed Asif Ali Shah ${ }^{1}$ \\ 1 Department of Mathematics, GC University Faisalabad Layyah Campus, Layyah 31200, Pakistan; \\ riasatyasin@gmail.com (R.A.); asifalishah695@gmail.com (S.A.A.S.) \\ 2 Division of Human Support System, Faculty of Symbiotic Systems Science, Fukushima University, \\ Fukushima 960-1296, Japan \\ * Correspondence: bamba@sss.fukushima-u.ac.jp
}

Received: 10 April 2019; Accepted: 26 April 2019; Published: 5 May 2019

\begin{abstract}
We investigate the massive vector field equation with the WKB approximation. The tunneling mechanism of charged bosons from the gauged super-gravity black hole is observed. It is shown that the appropriate radiation consistent with black holes can be obtained in general under the condition that back reaction of the emitted charged particle with self-gravitational interaction is neglected. The computed temperatures are dependant on the geometry of black hole and quantum gravity. We also explore the corrections to the charged bosons by analyzing tunneling probability, the emission radiation by taking quantum gravity into consideration and the conservation of charge and energy. Furthermore, we study the quantum gravity effect on radiation and discuss the instability and stability of black hole.
\end{abstract}

Keywords: higher dimension gauged super-gravity black hole; quantum gravity; quantum tunneling phenomenon; Hawking radiation

\section{Introduction}

General relativity is associated with the thermodynamics and quantum effect which are strongly supportive of each other. A black hole $(\mathrm{BH})$ is a compact object whose gravitational pull is so intense that can not escape the light. It was proved by Hawking that a $\mathrm{BH}$ has an additional property of emitting radiation. Since Hawking's great contribution on $\mathrm{BH}$ thermodynamics, the radiation from the $\mathrm{BH}$ has attained the attention of many researchers. There are many different process to obtain the Hawking radiation by applying the quantum field equations or the semi-classical phenomena. Different accesses to quantum gravity, as well as $\mathrm{BH}$ physics predict a minimum measure length or a maximum evident momentum and associated modifications of the principle of the Heisenberg uncertainty which is called the generalized uncertainty principle (GUP).

The thermal radiation coming from any stationary metric are calculated [1]. The physical image is that the radiation develops in the quasiclassical tunneling of particles from a gravitational barrier. They obtained a thermal spectrum and twice the temperature for Hawking radiation of non-rotating $\mathrm{BH}$. The expression $\exp \left(-2 \operatorname{Im}\left(\int p d r\right)\right)$ is not invariant under canonical transformation in generally and expressed that this implies half the correct temperature for $\mathrm{BH}$ [2]. In the setting of black rings significance, the radiation of the Dirac particles can be calculated by applying the Dirac wave equation in both the charged and uncharged case. The formulate of the field equations of uncharged and charged Dirac particles by using the covariant Dirac wave equation [3]. E. T. Akhmedov et al. [4] calculated Hawking radiation by using the quasi-classical phenomenon. The temporal contribution to gravitational WKB-like calculations are discussed in [5]. The authors analyzed that the quasiclassical 
method for gravitational backgrounds contains subtleties not found in the usual quantum mechanical tunneling problem.

V. Akhmedova et al. [6] compared the anomaly method and the WKB/tunneling method for finding radiation through non-trivial space-time. They conclude that these both method are not valid for all types of metrics. The discreteness space effect of the GUP are investigated in space [7]. Corda [8] analyzed interferometric detection of gravitational waves: the definitive test for general relativity. He conclude that accurate angular and frequency dependent response functions of interferometers for gravitational waves arising from various theories of gravity will be the definitive test for general relativity. The authors investigated insights and possible resolution to the information loss paradox via the tunneling picture [9]. They observe that the quantum correction give zero temperature for the radiation as the mass of the $\mathrm{BH}$ is zero.

From $\mathrm{F}(\mathrm{R})$ theory to Lorentz non-invariant models in modified gravity are investigated as [10]. The extended theories of gravity are discussed in [11]. The authors analyzed the problems of gravitational waves and neutrino oscillations through extended gravity theory. The authors [12] examined the rule to all alternative gravities, a particularly significance of scalar-tensor and $f(R)$ theories. Yale [13] analyzed the exact Hawking radiation of scalars, fermions and bosons 1-spin particles applying quantum tunneling phenomena without back reaction. The different dark energy models like $\Lambda$ cold dark matter, Pseudo-Rip and Little Rip universes, non-singular dark energy universes, the quintessence and phantom cosmologies with different types are analyzed [14].

Sharif and Javed [15] analyzed the Hawking radiation of fermion particles applying quantum tunneling phenomena from traversable wormholes. Corda [16] studied the important issue that the non-strictly continuous character of the Hawking radiation spectrum generates a natural correspondence between Hawking radiation and quasi-normal modes BH. Jan and Gohar [17] examined the Hawking temperature by quantum tunneling of scalars particles applying Klein-Gordon equation in WKB approximation. Kruglov [18] calculated the Hawking radiation by quantum tunneling of vector particles of BHs in 2 dimension applying Proca equation in WKB approximation. Matsumoto et al. [19] analyzed the time evolution of a thin black ring via Hawking radiation.

The different writers [20] determined the Hawking temperature by Hamilton-Jacobi equation of vector particles of Kerr and Kerr-Newman BHs by applying Proca and Lagrangian equations in WKB approximation. Corda [21] analyzed precise model of Hawking radiation from the tunneling mechanism and he found that pre-factor of the Parikh and Wilczek probability of emission depends on the BH quantum level. Anacleto [22] analyzed the GUP in the tunneling phenomena through Hamilton-Jacobi process to find the corrected temperature and entropy for three-dimensional noncommutative acoustic BHs. Anacleto et al. [23] studied the Hawking temperature by the Hamilton-Jacobi equation of spin $\frac{3}{2}$-particles of accelerating BHs, applying the Rarita-Schwinger equation in the WKB approximation. Chen and Huang [24] determined the Hawking temperature by quantum tunneling phenomena of vector particles of Vaidya BHs in applying the Proca equation in WKB approximation. Anacleto et al. [25] examined the quantum-corrected of self-dual BH entropy in tunneling phenomena with GUP. $\mathrm{Li}$ and $\mathrm{Zu}$ [26] analyzed the tunneling phenomena by the Hamilton-Jacobi equation of scalar particles of Gibbons-Maeda-Dilation BHs, applying the Klein-Gordon equation in the WKB approximation. Feng et al. [27] calculated the tunneling phenomena by the Hamilton-Jacobi equation of scalar particles of 4D and 5D BHs, applying the Proca equation in the WKB approximation. Saleh et al. [28] studied the Hawking radiation of 5D Lovelock $\mathrm{BH}$ with the Hamilton-Jacobi equation by using the Klein-Gordon equation. 
The authors [29] analyzed the cosmology of inflation by modifying terms of gravity and inflation in $\mathrm{F}(\mathrm{R})$ gravity. In the $\mathrm{F}(\mathrm{R})$ gravity, the Starobinsky inflation is discussed with the geometry of gravitational theories to the inflationary models. Övgun and Jusufi [30] calculated the tunneling phenomena by Hamilton-Jacobi process in a Lagrangian equation of spin-1 massive particle noncommutative BHs. Jusufi and Övgun [31] examined the Hawking temperature of vector and scalar particles from 5D Myers-Perry BHs and solved the Proca and Klein-Gordon equations by using the WKB approximation and Hamilton-Jacobi process in these cases.

The cosmological solutions, $\mathrm{BH}$ solutions and spherically symmetric developing through $\mathrm{F}(\mathrm{T})$ gravity were discussed in different cosmic expansion eras [32]. Singh et al. [33] discussed the Hawking temperature of vector particles from Kerr-Newman BHs in the Proca equation by applying the WKB approximation in the Hamilton-Jacobi process. Jusufi and Övgun [34] examined the Hawking radiation of massive particles from rotating charged black strings. Li and Zhao [35] calculated the tunneling process of massive particles from the neutral rotating anti-de Sitter BHs using the Proca wave equation in the WKB approximation. The different authors [36,37] determined the temperature of massive vector particles from the different types BHs by using tunneling phenomena. The nutshell, bounce, late time evolution and inflation were studied through modified gravity theories [38]. The future of gravitational theories in the framework of gravitational wave in astronomy was analyzed in [39]. The charged vector particles tunneling from black ring and 5D BH [40] is studied by wave equation to calculate the tunneling phenomena for charged particles as well as Hawking temperature. In this article, the authors have calculated the tunneling probability/rate and Hawking temperature for charged boson particles tunneling from horizon.

This paper is organization as follows: in Section 2 we discuss the tunneling rate and Hawking temperature of charged vector $W^{ \pm}$boson particles for $4 \mathrm{D}$ gauged super-gravity $\mathrm{BH}$ and also calculate quantum corrected tunneling probability and Hawking temperature. Section 3 is based on the analysis of for 7D gauged super-gravity BH. In Section 4, we discuss the graphical behavior of radiation for these types of BHs and visualize the stable and unstable state of BHs. In Section 5 we explain the conclusions and discussion.

\section{4-Dimension Gauged Super-Gravity Black Holes}

The super-gravity theory defined gauged theory through which the gauge boson, the super-partner of the particle is charged in some internal gauge group. Moreover, this theory is more important as compared to the ungauged case, therefore this theory has a negative cosmological constant $(\Lambda)$, where $\Lambda$ is stated in an anti-de Sitter BH. Now, for the study of a boson particle tunneling process form a BH in $(3+1)$ dimension theory of gauged super-gravity, we calculate the Hawking temperature of BH by tunneling phenomena at event horizon. The solution of $\mathrm{BH}$ occur in $D=4 \mathrm{~N}=8$ theory of gauged super-gravity (symmetry) [41]. The metric for such theory is given by [41]

$$
d s^{2}=-\left(H_{1} H_{2} H_{3} H_{4}\right)^{-\frac{1}{2}} f d t^{2}+\left(H_{1} H_{2} H_{3} H_{4}\right)^{\frac{1}{2}}\left(f^{-1} d r^{2}+r^{2} d \Omega_{2, k}^{2}\right),
$$

where $g=1 / L$ and $L$ is related to the cosmological constant $\Lambda=-3 g^{2}=-3 / L^{2}$ and the $\mu$ represent the non-extremality parameter [42]

$$
f=k-\frac{\mu}{r^{2}}+g^{2} r^{2} H_{1} H_{2} H_{3} H_{4}, \quad H_{i}=\frac{q_{i}}{r^{2}}+1, \quad(\text { for } i=1,2,3,4) .
$$

for radius $k=1$ and $k=0$, then $d \Omega_{2, k}^{2}$ represents the metrics on $\mathbf{S}^{2}$ and $\mathbf{R}^{2}$ respectively. The four electric potentials $A_{\mu}^{i}$ are defined as;

$$
A_{0}^{i}=\frac{\tilde{q}_{i}}{r^{2}+q_{i}}(\text { for } i=1,2,3,4)
$$


where $q_{i}$ and $\tilde{q}_{i}$ represent charges and physical charges of a BH. The line element from Equation (1) can be rewritten as

$$
d s^{2}=-F(r) d t^{2}+L^{-1}(r) d r^{2}+M(r) d \theta^{2}+N(r) d \phi^{2},
$$

where

$$
\begin{array}{ll}
F(r)=f\left(H_{1} H_{2} H_{3} H_{4}\right)^{-\frac{1}{2}} & L^{-1}(r)=f^{-1}\left(H_{1} H_{2} H_{3} H_{4}\right)^{\frac{1}{2}} \\
M(r)=r^{2}\left(H_{1} H_{2} H_{3} H_{4}\right)^{\frac{1}{2}} & N(r)=r^{2} \sin ^{2} \theta\left(H_{1} H_{2} H_{3} H_{4}\right)^{\frac{1}{2}} .
\end{array}
$$

The wave equation of motion comprises of GUP obtained from the Glashow-Weinberg-Salam model $[20,43]$

$$
\begin{aligned}
& \partial_{\mu}\left(\sqrt{-g} \Phi^{v \mu}\right)+\sqrt{-g} \frac{m^{2}}{h^{2}} \Phi^{v}+\sqrt{-g} \frac{i}{h} e A_{\mu} \Phi^{v \mu}+\sqrt{-g} \frac{i}{h} e F^{v \mu} \Phi_{\mu}+\alpha \hbar^{2} \partial_{0} \partial_{0} \partial_{0} \\
& \left(\sqrt{-g} g^{00} \Phi^{0 v}\right)-\alpha \hbar^{2} \partial_{i} \partial_{i} \partial_{i}\left(\sqrt{-g} g^{i i} \Phi^{i v}\right)=0,
\end{aligned}
$$

where $g$ is a determinant coefficient matrix, $\Phi^{\mu v}$ is anti-symmetric tensor and $m$ is particles mass, since

$$
\begin{aligned}
\Phi_{v \mu} & =\left(1-\alpha \hbar^{2} \partial_{v}^{2}\right) \partial_{\nu} \Phi_{\mu}-\left(1-\alpha \hbar^{2} \partial_{\mu}^{2}\right) \partial_{\mu} \Phi_{v}+\left(1-\alpha \hbar^{2} \partial_{v}^{2}\right) \frac{l}{\hbar} e A_{\nu} \Phi_{\mu} \\
& -\left(1-\alpha \hbar^{2} \partial_{\mu}^{2}\right) \frac{l}{\hbar} e A_{\mu} \Phi_{\nu}
\end{aligned}
$$

where $\alpha, A_{\mu}, e$ and $\triangle_{\mu}$ are the quantum gravity parameter (dimensionless positive parameter), vector potential of the charged $\mathrm{BH}$, the charge of the particle and covariant derivative, respectively. As the wave equations for the $W^{+}$and $W^{-}$boson particles are alike, the tunneling actions should be alike too $\left(W^{+}=-W^{-}\right)$. We will view the $W^{+}$boson particle case after simplification and the results of such case can be changed to multiply negative sign $W^{-}$boson particles due to the digitalization of the metric. There value of $\Phi^{\mu}$ and $\Phi^{v \mu}$ are given by

$$
\begin{aligned}
\Phi^{0} & =\frac{\Phi_{0}}{F(r)}, \quad \Phi^{1}=\frac{\Phi_{1}}{L^{-1}(r)}, \quad \Phi^{2}=\frac{\Phi_{2}}{M(r)}, \quad \Phi^{3}=\frac{\Phi_{3}}{N(r)}, \\
\Phi^{01} & =\frac{\Phi_{01}}{F(r) L^{-1}(r)}, \quad \Phi^{02}=\frac{\Phi_{02}}{F(r) M(r)}, \quad \Phi^{03}=\frac{\Phi_{03}}{F(r) N(r)}, \\
\Phi^{12} & =\frac{\Phi_{12}}{L^{-1}(r) M(r)}, \quad \Phi^{13}=\frac{\Phi_{13}}{L^{-1}(r) N(r)}, \quad \Phi^{23}=\frac{\Phi_{23}}{M(r) N(r)} .
\end{aligned}
$$

The WKB approximation is given in [44], i.e.,

$$
\Phi_{v}=c_{v} \exp \left[\frac{i}{\hbar} \oplus_{0}(t, r, \theta, \phi)+\sum_{i=1}^{i=n} \hbar^{i} \oplus_{i}(t, r, \theta, \phi)\right] .
$$


Substituting the Equation (4) into the wave Equation (3), where $i=1,2,3, \ldots$ neglecting the terms. We get the set of equations below:

$$
\begin{aligned}
& L(r)\left[c_{1}\left(\partial_{0} \oplus_{0}\right)\left(\partial_{1} \oplus_{0}\right)+c_{1} \alpha\left(\partial_{0} \oplus_{0}\right)^{3}\left(\partial_{1} \oplus_{0}\right)-c_{0}\left(\partial_{1} \oplus_{0}\right)^{2}-c_{0}\left(\partial_{1} \oplus_{0}\right)^{4} \alpha\right. \\
& \left.+e A_{0} c_{1}\left(\partial_{1} \oplus_{0}\right)+e A_{0} c_{1} \alpha\left(\partial_{1} \oplus_{0}\right)\left(\partial_{0} \oplus_{0}\right)^{2}\right]+\frac{1}{M(r)}\left[c_{2}\left(\partial_{0} \oplus_{0}\right)\left(\partial_{2} \oplus_{0}\right)+\alpha c_{2}\right. \\
& \left(\partial_{0} \oplus_{0}\right)^{3}\left(\partial_{2} \oplus_{0}\right)-c_{0}\left(\partial_{2} \oplus_{0}\right)^{2}-\alpha c_{0}\left(\partial_{2} \oplus_{0}\right)^{4}+e A_{0} c_{2}\left(\partial_{2} \oplus_{0}\right)+\alpha e A_{0} c_{2}\left(\partial_{0} \oplus_{0}\right)^{2} \\
& \left.\left(\partial_{2} \oplus_{0}\right)\right]+\frac{1}{N(r)}\left[c_{3}\left(\partial_{0} \oplus_{0}\right)\left(\partial_{3} \oplus_{0}\right)+\alpha c_{3}\left(\partial_{0} \oplus_{0}\right)^{3}\left(\partial_{3} \oplus_{0}\right)+c_{0}\left(\partial_{3} \oplus_{0}\right)^{2}\right. \\
& \left.+\alpha c_{0}\left(\partial_{3} \oplus_{0}\right)^{4}+e A_{0} c_{3}\left(\partial_{3} \oplus_{0}\right)+\alpha c_{3} e A_{0}\left(\partial_{0} \oplus_{0}\right)^{2}\left(\partial_{3} \oplus_{0}\right)\right]-m^{2} c_{0}=0 \\
& \frac{-1}{F(r)}\left[c_{0}\left(\partial_{0} \oplus_{0}\right)\left(\partial_{1} \oplus_{0}\right)+c_{0} \alpha\left(\partial_{0} \oplus_{0}\right)\left(\partial_{1} \oplus_{0}\right)^{3}-c_{1}\left(\partial_{0} \oplus_{0}\right)^{2}-c_{1} \alpha\left(\partial_{0} \oplus_{0}\right)^{4}\right. \\
& \left.-e A_{0} c_{1}\left(\partial_{0} \oplus_{0}\right)-\alpha e A_{0} c_{1}\left(\partial_{1} \oplus_{0}\right)^{2}\left(\partial_{0} \oplus_{0}\right)\right]+\frac{1}{M(r)}\left[c_{2}\left(\partial_{1} \oplus_{0}\right)\left(\partial_{2} \oplus_{0}\right)\right. \\
& \left.+\alpha c_{2}\left(\partial_{1} \oplus_{0}\right)^{3}\left(\partial_{2} \oplus_{0}\right)-c_{1}\left(\partial_{2} \oplus_{0}\right)^{2}-\alpha c_{1}\left(\partial_{2} \oplus_{0}\right)^{4}\right]+\frac{1}{N(r)}\left[c_{3}\left(\partial_{1} \oplus_{0}\right)\left(\partial_{3} \oplus_{0}\right)\right. \\
& \left.+c_{3} \alpha\left(\partial_{1} \oplus_{0}\right)^{3}\left(\partial_{3} \oplus_{0}\right)-c_{1}\left(\partial_{3} S_{0}\right)^{2}-c_{1} \alpha\left(\partial_{3} \oplus_{0}\right)^{4}\right]-m^{2} c_{1}-\frac{1}{F} e A_{0} \\
& {\left[c_{0}\left(\partial_{1} \oplus_{0}\right)+\alpha c_{0}\left(\partial_{1} \oplus_{0}\right)^{3}-c_{1}\left(\partial_{0} \oplus_{0}\right)-\alpha c_{1}\left(\partial_{0} \oplus_{0}\right)^{3}-c_{1} e A_{0}\right.} \\
& \left.-e A_{0} \alpha c_{1}\left(\partial_{1} \oplus_{0}\right)^{2}\right]=0 \\
& \frac{1}{F(r)}\left[c_{0}\left(\partial_{0} \oplus_{0}\right)\left(\partial_{2} \oplus_{0}\right)+\alpha c_{0}\left(\partial_{0} \oplus_{0}\right)\left(\partial_{2} \oplus_{0}\right)^{3}-c_{2}\left(\partial_{0} \oplus_{0}\right)^{2}-\alpha c_{2}\left(\partial_{0} \oplus_{0}\right)^{4}\right. \\
& \left.-e A_{0}\left(\partial_{0} \oplus_{0}\right) c_{2}-e A_{0}\left(\partial_{0} \oplus_{0}\right)^{3} c_{2} \alpha\right]-\frac{1}{L^{-1}(r)}\left[c_{2}\left(\partial_{1} \oplus_{0}\right)^{2}+\alpha c_{2}\left(\partial_{1} \oplus_{0}\right)^{4}\right. \\
& \left.-c_{1}\left(\partial_{1} \oplus_{0}\right)\left(\partial_{2} \oplus_{0}\right)-\alpha c_{1}\left(\partial_{1} \oplus_{0}\right)\left(\partial_{2} \oplus_{0}\right)^{3}\right]+\frac{1}{N(r)}\left[c_{3}\left(\partial_{2} \oplus_{0}\right)\left(\partial_{3} \oplus_{0}\right)\right. \\
& \left.+\alpha c_{3}\left(\partial_{2} \oplus_{0}\right)^{3}\left(\partial_{3} \oplus_{0}\right)-c_{2}\left(\partial_{3} \oplus_{0}\right)^{2}-\alpha c_{2}\left(\partial_{3} \oplus_{0}\right)^{4}\right]-\frac{e A_{0}}{F(r)}\left[c_{0}\left(\partial_{2} \oplus_{0}\right)\right. \\
& \left.+\alpha c_{0}\left(\partial_{2} \oplus_{0}\right)^{3}-c_{2}\left(\partial_{0} \oplus_{0}\right)-\alpha c_{2}\left(\partial_{0} \oplus_{0}\right)^{3}+c_{2} e A_{0}+\alpha c_{2} e A_{0}\left(\partial_{0} \oplus_{0}\right)^{2}\right] \\
& -m^{2} c_{2}=0 \\
& \frac{1}{F(r)}\left[c_{0}\left(\partial_{0} \oplus_{0}\right)\left(\partial_{3} \oplus_{0}\right)+\alpha c_{0}\left(\partial_{0} \oplus_{0}\right)\left(\partial_{3} \oplus_{0}\right)^{3}-c_{3}\left(\partial_{0} \oplus_{0}\right)^{2}-\alpha c_{3}\left(\partial_{0} \oplus_{0}\right)^{4}\right. \\
& \left.-e A_{0}\left(\partial_{0} \oplus_{0}\right) c_{3}-e A_{0}\left(\partial_{3} \oplus_{0}\right)^{2}\left(\partial_{0} \oplus_{0}\right) c_{3} \alpha\right]+\frac{1}{L^{-1}(r)}\left[c_{3}\left(\partial_{1} \oplus_{0}\right)^{2}+\alpha c_{3}\left(\partial_{1} \oplus_{0}\right)^{4}\right. \\
& \left.-c_{1}\left(\partial_{3} \oplus_{0}\right)\left(\partial_{1} \oplus_{0}\right)-\alpha c_{1}\left(\partial_{1} \oplus_{0}\right)\left(\partial_{3} \oplus_{0}\right)^{3}\right]+\frac{1}{M(r)}\left[c_{3}\left(\partial_{2} \oplus_{0}\right)^{2}\right. \\
& \left.+\alpha c_{3}\left(\partial_{2} \oplus_{0}\right)^{4}-c_{2}\left(\partial_{2} \oplus_{0}\right)\left(\partial_{3} \oplus_{0}\right)-\alpha c_{2}\left(\partial_{3} \oplus_{0}\right)^{3}\left(\partial_{2} \oplus_{0}\right)\right]+\frac{e A_{0}}{F(r)}\left[c_{0}\left(\partial_{3} \oplus_{0}\right)\right. \\
& \left.+\alpha c_{0}\left(\partial_{3} \oplus_{0}\right)^{3}-c_{3}\left(\partial_{0} \oplus_{0}\right)-\alpha c_{3}\left(\partial_{0} \oplus_{0}\right)^{3}-c_{3} e A_{0}-\alpha c_{3} e A_{0}\left(\partial_{3} \oplus_{0}\right)^{2}\right] \\
& -m^{2} c_{3}=0 \text {. }
\end{aligned}
$$

We can choose the separation of variables,

$$
\oplus_{0}=-(E-j \Omega) t+W(r)+j \phi+v(\theta),
$$


where $j, E$ and $\Omega$ represent angular momentum, energy and angular velocity of particle, respectively. Here, $W(r)$ and $v(\theta)$ are two arbitrary functions. The matrix equation can be obtain from the Equations (5)-(8),

$$
K\left(c_{0}, c_{1}, c_{2}, c_{3}\right)^{T}=0,
$$

which gives " $K$ " is a order of ' $4 \times 4^{\prime}$ matrix and its components are given by:

$$
\begin{aligned}
& K_{00}=\frac{\dot{W}^{2}+\alpha \dot{W}^{4}}{L^{-1}(r)}-\frac{j^{2}+\alpha j^{4}}{M(r)}+\frac{\dot{v}^{3}+\alpha \dot{v}^{4}}{N(r)}-m^{2}, \\
& K_{01}=-\frac{\dot{W}(E-j \Omega)+\alpha \dot{W}(E-j \Omega)^{3}}{L^{-1}(r)}+\frac{\dot{W} e A_{0}+\alpha \dot{W} e A_{0}(E-j \Omega)^{2}}{L^{-1}(r)}, \\
& K_{02}=-\frac{(E-j \Omega) j+\alpha(E-j \Omega) j}{M(r)}+\frac{e A_{0} j+\alpha(E-j \Omega)^{2} e A_{0} j}{M(r)}, \\
& K_{03}=-\frac{\dot{v}(E-j \Omega)+\alpha \dot{v}(E-j \Omega)^{3}}{N(r)}+\frac{e A_{0} \dot{v}+\alpha e A_{0} \dot{v}(E-j \Omega)^{2}}{N(r)}, \\
& K_{10}=\frac{(E-j \Omega) \dot{W}+\alpha(E-j \Omega) \dot{W}^{3}}{F(r)}-\frac{e A_{0} \dot{W}+\alpha e A_{0} \dot{W}^{3}}{F(r)}, \\
& K_{11}=\frac{(E-j \Omega)^{2}+\alpha(E-j \Omega)^{4}}{F(r)}+\frac{(E-j \Omega) e A_{0}-\alpha \dot{W}(E-j \Omega) e A_{0}}{F(r)} \\
& -\frac{j^{2}-\alpha j^{4}}{M(r)}-\frac{\dot{v}^{2}-\alpha \dot{v}^{4}}{N(r)}-m^{2}-\frac{1}{F(r)} e A_{0}\left[(E-j \Omega)+\alpha(E-j \Omega)^{3}\right. \\
& \left.-e A_{0}-\alpha e A_{0} \dot{W}^{2}\right] \\
& K_{12}=\frac{\dot{W} j+\alpha \dot{W}^{3} j}{M(r)}, \quad K_{13}=\frac{\dot{v} \dot{W}+\dot{v} \alpha \dot{W}^{3}}{N(r)}, \\
& K_{20}=-\frac{j(E-j \Omega)+\alpha j^{3}(E-j \Omega)}{F(r)}-e A_{0} \frac{j+\alpha j^{3}}{F(r)}, K_{21}=\frac{\dot{W} j+\alpha \dot{W} j^{3}}{L^{-1}(r)}, \\
& K_{22}=-\frac{1}{F(r)}\left[-(E-j \Omega)^{2}-\alpha(E-j \Omega)^{4}+e A_{0}(E-j \Omega)+e A_{0} \alpha(E-j \Omega)^{3}\right] \\
& -\frac{\dot{W}^{2}+\alpha \dot{W}^{4}}{L^{-1}(r)}-\frac{\dot{v}^{2}+\alpha \dot{v}^{4}}{N(r)}-m^{2}, K_{23}=\frac{j \dot{v}+\alpha j^{3} \dot{v}}{N(r)}, \\
& K_{30}=\frac{-1}{F(r)}\left[(E-j \Omega) \dot{v}+\alpha(E-j \Omega) \dot{v}^{3}\right]+\frac{e A_{0} \dot{v}+e A_{0} \alpha \dot{v}^{3}}{F(r)}, \\
& K_{31}=\frac{-\dot{W} \dot{v}-\alpha \dot{W} \dot{v}^{3}}{L^{-1}(r)}, K_{32}=\frac{-j \dot{v}-\alpha j \dot{v}^{3}}{M(r)}, \\
& K_{33}=-\frac{1}{F(r)}\left[(E-j \Omega)^{2}+\alpha(E-j \Omega)^{4}-(E-j \Omega) e A_{0}-\alpha(E-j \Omega) e A_{0} \dot{v}^{3}\right]+ \\
& \frac{\dot{W}^{2}+\alpha \dot{W}^{4}}{L^{-1}(r)}-\frac{j^{2}+\alpha j^{4}}{M(r)}+\frac{e A_{0}}{F(r)}\left[(E-j \Omega)+\alpha(E-j \Omega)^{3}-e A_{0}-\alpha e A_{0} \dot{v}^{3}\right] \\
& -m^{2}
\end{aligned}
$$

where $\dot{W}=\partial_{r} \oplus_{0}, \dot{v}=\partial_{\theta} \oplus_{0}$ and $j=\partial_{\phi} \oplus_{0}$. The non-trivial solution is $|\mathbf{K}|=0$ and solving these equations yields:

$$
i m W^{ \pm}= \pm \int \sqrt{\frac{\left(E-e A_{0}-j \Omega\right)^{2}+X_{1}\left(1+\frac{X_{2}}{X_{1}} \alpha\right)}{L(r)}} d r
$$

where - and + denote the incoming and outgoing particles, respectively. The function ' $X_{1}^{\prime}$ can be defined as $X_{1}=\frac{j^{2}}{M(r)}$ and $X_{2}=\frac{\alpha(E-j \Omega)^{4}}{F(r)}-\frac{\alpha(E-j \Omega) e A_{0} \dot{v}^{3}}{F(r)}-\frac{\alpha \dot{W}^{4}}{L^{-1}(r)}+\alpha \frac{j^{4}}{M(r)}-\frac{e A_{0}}{F(r)}\left[\alpha(E-j \Omega)^{3}-\right.$ 
$\left.\alpha e A_{0} \dot{v}^{3}\right]+m^{2}$ represent the angular velocity at the event horizon. Integrating Equation (10) around the pole, we get

$$
i m W^{ \pm}= \pm i \pi \frac{\left(E-A_{0} e-j \Omega\right)}{2 \kappa\left(r_{+}\right)}(1+\Xi \alpha),
$$

and the surface gravity of the $4 \mathrm{D}$ gauged super-gravity $\mathrm{BH}$ [41] is given by

$$
\kappa\left(r_{+}\right)=\frac{3 r_{+}^{4}+2 r_{+}^{3} q_{1} q_{2} q_{3} q_{4}+r_{+}^{2}\left(\sum_{i<j}^{4} q_{i} q_{j}+1\right)-q_{1} q_{2} q_{3} q_{4}}{2 r_{+} \sqrt{\prod_{i=1}^{4}\left(r_{+}+q_{i}\right)}} .
$$

The tunneling probability $\Gamma\left(i m W^{+}\right)$for boson vector particles is given by

$$
\begin{aligned}
\Gamma\left(i m W^{+}\right) & =\frac{\operatorname{Prob}[\text { emission }]}{\text { Prob }[\text { absorption }]}=\frac{\exp \left[-2\left(i m W^{+}+i m v\right)\right]}{\exp \left[-2\left(i m W^{-}-i m v\right)\right]}=\exp \left[-4 i m W^{+}\right] \\
& =\exp \left[-\pi \frac{\left(E-e A_{0}-j \Omega\right) r_{+} \sqrt{\prod_{i=1}^{4}\left(r_{+}+q_{i}\right)}}{3 r_{+}^{4}+2 r_{+}^{3} q_{1} q_{2} q_{3} q_{4}+r_{+}^{2}\left(\sum_{i<j}^{4} q_{i} q_{j}+1\right)-q_{1} q_{2} q_{3} q_{4}}\right] \\
& \times(1+\Xi \alpha) .
\end{aligned}
$$

The particles that tunnel outside the event horizon will fall into the $\mathrm{BH}$, and one has $\operatorname{Prob}[$ emission $]=1$ then $i m W^{-}-i m v=0$.

Now, we can calculate the $T_{H}\left(i m W^{+}\right)$by comparing the $\Gamma\left(i m W^{+}\right)$with the Boltzmann formula $\Gamma_{B}\left(i m W^{+}\right) \approx e^{-\left(E-e A_{0}-j \Omega\right) / T_{H}\left(i m W^{+}\right)}$, we get

$$
\begin{aligned}
T_{H}\left(i m W^{+}\right) & =\frac{3 r_{+}^{4}+2 r_{+}^{3} q_{1} q_{2} q_{3} q_{4}+r_{+}^{2}\left(\sum_{i<j}^{4} q_{i} q_{j}+1\right)-q_{1} q_{2} q_{3} q_{4}}{4 \pi r_{+} \sqrt{\prod_{i=1}^{4}\left(r_{+}+q_{i}\right)}} \\
& \times(1+\Xi \alpha)^{-1} .
\end{aligned}
$$

The $\Gamma\left(i m W^{+}\right)$depends on the radial coordinate at the outer horizon $r_{+}, A_{0}$ vector potentials, $E$ energy, $j$ angular momentum, $e$ charge of particles, $q_{i}$ charge of a $4 \mathrm{D}$ gauged super-gravity BHs, $\alpha$ quantum gravity and $\Omega$ represent the angular velocity on this horizon.

\section{5-Dimension Gauged Super-Gravity Black Holes}

This BH solution occurs for $N=8, D=5$, in gauged super-gravity theory (symmetry) [41]. Now, a particular case is discussed, where the solution was developed (STU-model) for the results of $N=2, D=5$, gauged super-gravity theory wave equation of motion. The line element for $5 D \mathrm{BH}$ in the theory of gauged super-gravity is given as [41]

$$
d s^{2}=-f\left(H_{3} H_{2} H_{1}\right)^{-\frac{2}{3}} d t^{2}+f^{-1}\left(H_{3} H_{2} H_{1}\right)^{\frac{1}{3}} d r^{2}+\left(H_{3} H_{2} H_{1}\right)^{\frac{1}{3}} r^{2} d \Omega_{3, k}^{2}
$$

where

$$
f=g^{2} r^{2} H_{3} H_{2} H_{1}-\frac{\mu}{r^{2}}+k, \quad H_{i}=1+\frac{q_{i}}{r^{2}},
$$

here $\mathrm{i}=1,2,3$ and for radius $k=1$ and $k=0$, then $d \Omega_{3, k}^{2}$ represents the metrics on $\mathbf{S}^{3}$ and $\mathbf{R}^{3}$ respectively. It is connected to $A D M$ mass i.e., $g=1 / L$, which indicates $A d S_{5}$ 's inverse radius and depends upon the cosmological constant, $\Lambda=-6 / L^{2}=-6 g^{2}$, and the $q_{i}$ are BH charges. The result of the wave equation is the form of the three gauge potential field $A_{\mu}^{i}$ from

$$
A_{0}^{i}=\frac{\tilde{q}_{i}}{q_{i}+r^{2}}
$$


here, $i=1,2,3$ and $\tilde{q}_{i}$ are BH physical charges. It is observed that Gauss's theorem is applicable for these charges. The corrected temperature $\left(T_{H}^{\prime}\right)$ can be calculated as

$$
T_{H}^{\prime}\left(i m W^{+}\right)=\frac{\left(\Sigma_{i=1}^{3} q_{i}+1\right) r_{+}^{4}-\prod_{i=1}^{3} q_{i}+2 r_{+}^{6}}{2 \pi r_{+}^{2} \sqrt{\prod_{i=1}^{3}\left(q_{i}+r_{+}^{2}\right)}}(1+\Xi \alpha)^{-1} .
$$

The corrected tunneling rate depends on energy $(E)$, potential $\left(A_{0}\right)$, angular momentum $\left(\Omega_{H}\right)$, the outer horizon $\left(r_{+}\right)$radial coordinate, correction parameter $(\alpha)$ and BH charge $\left(q_{i}\right)$. We notice that the corrected temperature of boson particles denoted by Equation (17) is same as $(\alpha=0)$, the $5 D \mathrm{BH}$ temperature in the theory of gauged super-gravity in Equation (3.12) of Reference [43]. The $T_{H}\left(i m W^{+}\right)$ is related to the radial coordinate on the outer horizon $r_{+}, \alpha$ quantum gravity and charge $q_{i}$ of a $4 \mathrm{D}$ gauged super-gravity BHs respectively.

\section{7-Dimension Black Holes in Theory of Gauged Super-Gravity}

We calculate a boson particle's quantum tunneling spectrum from a $\mathrm{BH}$ in $7 \mathrm{D}$ gauged super-gravity theory and also determine the tunneling rate of boson particles and the corresponding temperature at $\mathrm{BH}$ outer horizon $r_{+}$. The solutions of $\mathrm{BH}$ occur when $D=7$ and $N=4$ in the gauged super-gravity theory (symmetry) [41]. Firstly, this result was developed in as a special case of solutions of cases when $D=7, N=4$ gauged super-gravity through the equations of motion. The metric of a $\mathrm{BH}$ in $7 \mathrm{D}$ gauged super-gravity theory is [41]

$$
d s^{2}=-\left(H_{1} H_{2}\right)^{-\frac{4}{5}} f d t^{2}+\left(H_{1} H_{2}\right)^{\frac{1}{5}}\left(f^{-1} d r^{2}+r^{2} d \Omega_{5, k}^{2}\right),
$$

where

$$
f=g^{2} r^{2} H_{1} H_{2}-\frac{\mu}{r^{4}}+k, \quad H_{i}=\frac{q_{i}}{r^{4}}+1, \quad(\text { for } i=1,2)
$$

where $g=1 / L=1$ and $L$ is related to the cosmological constant $\Lambda=-15 / L^{2}$. The two gauge field electric potentials $A_{\mu}^{i}$ through the result ofthe wave equation of motion are given by

$$
A_{0}^{i}=\frac{\tilde{q}_{i}}{r^{4}+q_{i}}(\text { for } i=1,2)
$$

The corresponding Hawking temperature at the horizon can be obtained as

$$
\check{T}\left(i m W^{+}\right)=\left[\frac{3 r_{+}^{8}+2 r_{+}^{6}+r_{+}^{4}\left(q_{1}+q_{2}\right)-q_{1} q_{2}}{\pi r_{+}^{3} \sqrt{\left(r_{+}^{4}+q_{1}\right)\left(r_{+}^{4}+q_{2}\right)}}\right](1+\Xi \alpha)^{-1} .
$$

The Hawking temperature depends on parameters $r_{0}, q_{2}$, and $q_{1}$.

\section{Graphical Analysis}

In this section, we describe the graphical behavior of quantum corrected Hawking temperature in Equations (14), (17) and (19) as shown in Figures 1-3, respectively, for arbitrary parameter $\Xi=1$ and also study the stable and unstable states of BHs. 


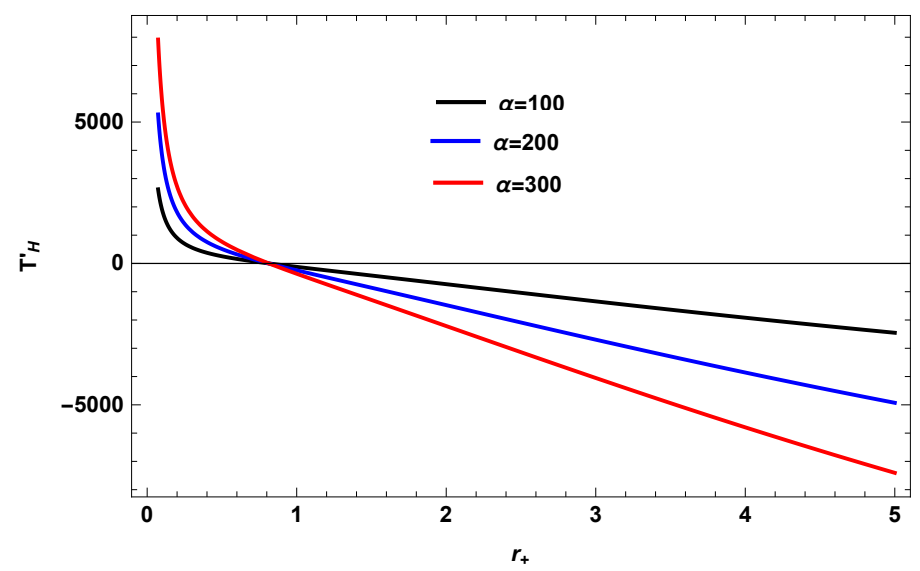

Figure 1. $T_{H}\left(i m W^{+}\right)$versus $r_{+}$for $q_{1}=q_{2}=q_{3}=q_{4}=0.5$ and $q_{1}=q_{2}=q_{3}=q_{4}=5$.

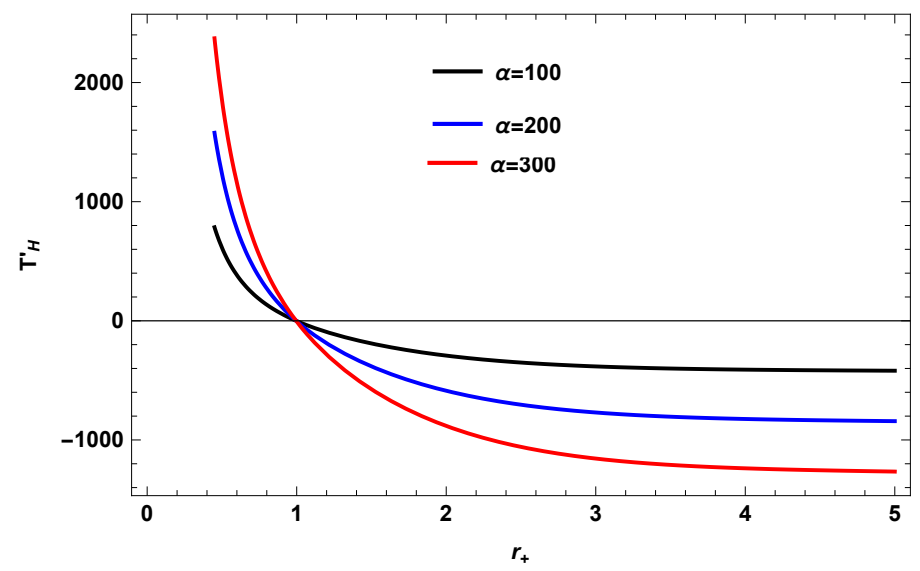

Figure 2. $T_{H}^{\prime}\left(i m W^{+}\right)$versus $r_{+}$for $q_{1}=q_{2}=q_{3}=0.5$ and $q_{1}=q_{2}=q_{3}=5$.

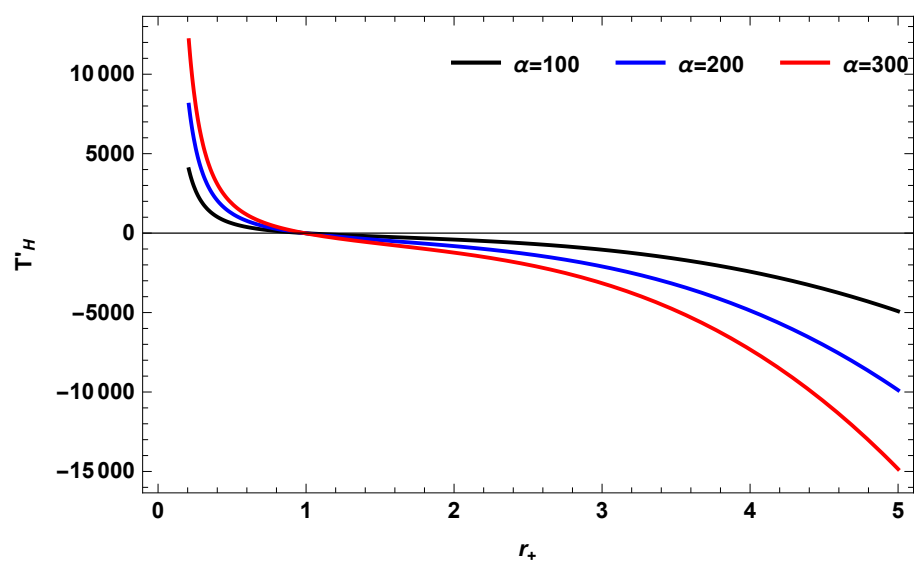

Figure 3. $\check{T}\left(\mathrm{imW} \mathrm{W}^{+}\right)$versus $r_{+}$for $q_{1}=q_{2}=0.5$ and $q_{1}=q_{2}=5$.

$T_{H}$ Versus $r_{+}$

In this subsection, we analyze the graphical behavior of corrected Hawking temperature $T_{H}$ w.r.t the horizon $r_{+}$for the $4 D, 5 D$ and $7 D$ gauged super-gravity BHs. Moreover, we study the physical significance of these graphs in the presence of correction parameter $\alpha$ and discuss the stable and unstable condition of corresponding BHs.

The $T_{H}\left(i m W^{+}\right)$slightly increase with increasing horizon and a slight change in the value of the correction parameter $\alpha=1$ can cause a small increase in temperature, but the non-physical behavior identifies the unstable state of BHs. 
In Figures 1-3, after initial increases in the particular range the temperature sharply increases with positive value. The non-physical behavior of the temperature increases with increasing horizon shows the instability of $\mathrm{BH}$.

\section{Conclusions and Discussion}

In summary, applying the Hamilton-Jacobi phenomena of the tunneling formalism, we have studied the metric of the four, five and seven dimensional gauged super-gravity BHs. For this aim, we applied the Lagrangian wave equation with the setting of electromagnetism to analyze the tunneling of a massive charged boson (1-spin) particles from four, five and seven dimensional gauged super-gravity BHs having charges and physical charges. In this paper, we have extended the work of massive vector particles tunneling probability/rate for more generalized BHs in four, five and seven dimensional spaces and also observed the Hawking temperatures at which the particles tunnel through horizons. We have applied the Lagrangian equation to study the tunneling probabilty/rate of massive boson particles from four, five and seven dimensional gauged super-gravity BHs. In the Lagrangian equation, we applied the WKB approximation and which implies to the set of field wave equations, then apply separation of variables to find these wave equations.

The radial part can be obtained by applying the matrix of coefficients, whose determinant is equal to zero. We have developed the tunneling probability and temperature for these BHs at the outer horizon using surface gravity. The tunneling and temperature depend on the setting parameters of the $\mathrm{BHs}$ and quantum gravity. It is worth to study that the back-reaction and self-gravitating effects of boson charged particles on these BHs have been ignored, the calculated temperature are the parameters of BHs and quantum gravity.

The significance of the BHs, for the all types of particles having charged and uncharged, the tunneling rate will be change by viewing their semi-classical phenomenon and corresponding temperatures must be same for all types of charged and uncharged particles. We analyzed the part of the action which is imaginary, the tunneling probability/rate and temperature were introduced by charged massive vector particles due to gravity near the outer horizon $r_{+}$. Moreover, for the correction to the energy and tunneling rate of the massive boson particle GUP was introduced near the outer horizon $r_{+}$in our computation. From our analysis, we have analyzed that the corrected temperature at which charged boson particles tunnel through the outer horizon $r_{+}$is independent of the dimension of a BHs, and temperature is dependent on parameters of a metric and quantum gravity. The corrected temperature is shown to depend on the quantum gravity effect $\alpha$. Both temperatures have the standard Hawking temperature limit when $(\alpha=0)$, then the GUP effect completely vanished.

From our analysis we also concluded that the temperature at which particles tunnel through the outer horizon $r_{+}$does not depend of the dimension of BHs in space. In particular the BH geometries, for the particles having different spin up and spin down the tunneling probabilities will be discovered to be the same by considering semi-classical phenomenon. Thus, their corresponding temperatures must be the same for all spin up and spin down particles. For these cases, we have carried out the calculations for more general BHs. Hence, the result still applies if the set $\mathrm{BH}$ parameters are more general.

- In the presence of charges, the $\mathrm{BH}$ was initially stable and attained a stability in a small domain and then becomes unstable till $r_{+} \rightarrow+\infty$.

- $\quad$ The 4D, 5D and 7D BHs remained stable and unstable in quantum gravity minima and maxima respectively.

- The 4D, 5D and 7D BHs in the theory of gauged super-gravity remains unstable in the presence of the charge and correction parameter $\alpha$.

Author Contributions: All authors contributed equally to this paper.

Funding: This research received no external funding. 
Acknowledgments: The work of KB was partially supported by the JSPS KAKENHI Grant Number JP 25800136 and Competitive Research Funds for Fukushima University Faculty (18RI009).

Conflicts of Interest: The authors declare no conflict of interest.

\section{References}

1. Akhmedov, E.T.; Akhmedova, V.; Singleton, D.; Pilling, T. Thermal radiation of various gravitational backgrounds. Int. J. Mod. Phys. A 2007, 22, 1705-1715. [CrossRef]

2. Chowdhury, B.D. Problems with tunneling of thin shells from black holes. Pramana 2008, 70, 3-26. [CrossRef]

3. Jiang, Q.Q. Dirac particle tunneling from black rings. Phys. Rev. D 2008, 78, 044009. [CrossRef]

4. Akhmedov, E.T.; Pilling, T.; Singleton, D. Subtleties in the quasiclassical calculation of Hawking radiation. Int. J. Mod. Phys. D 2008, 17, 2453-2458. [CrossRef]

5. Akhmedova, V.; Pilling, T.; Gill, A.D.; Singleton, D. Temporal contribution to gravitational WKB-like calculations. Phys. Lett. B 2008, 666, 269-271. [CrossRef]

6. Akhmedova, V.; Pilling, T.; Gill, A.D.; Singleton, D. Comments on anomaly versus WKB/tunneling methods for calculating Unruh radiation. Phys. Lett. B 2009, 673, 227-231. [CrossRef]

7. Ali, A.F.; Das, S.; Vagenas, E.C. Discreteness of Space from the Generalized Uncertainty Principle. Phys. Lett. B 2009, 678, 497-499. [CrossRef]

8. Corda, C. Interferometric detection of gravitational waves: The definitive test for General Relativity. Int. J. Mod. Phys. D 2009, 18, 2275-2282. [CrossRef]

9. Singleton, D.; Vagenas, E.C.; Zhu, T.; Ren, J.R. Insights and possible resolution to the information loss paradox via the tunneling picture. JHEP 2010, 1008, 089. [CrossRef]

10. Nojiri, S.; Odintsov, S.D. Unified cosmic history in modified gravity: from $F(R)$ theory to Lorentz non-invariant models. Phys. Rept. 2011, 505, 59-144. [CrossRef]

11. Capozziello, S.; Laurentis, M.D. Extended Theories of Gravity. Phys. Rept. 2011, 509, 167. [CrossRef]

12. Valerio, F.; Salvatore, C. Beyond Einstein Gravity: A survey of Gravitational Theories for Cosmology and Astrophysics; Fundamental Theories of Physics; Spring: New York, NY, USA, 2011.

13. Yale, A. Exact Hawking Radiation of Scalars, Fermions, and Bosons Using the Tunneling Method without Back-Reaction. Phys. Lett. B 2011, 697, 398-403. [CrossRef]

14. Bamba, K.; Capozziello, S.; Nojiri, S.; Odintsov, S.D. Dark energy cosmology: The equivalent description via different theoretical models and cosmography tests. Astrophys. Space Sci. 2012, 342, 155-228. [CrossRef]

15. Sharif, M.; Javed, W. Fermion tunneling for traversable wormholes. Can. J. Phys. 2013, 91, 43-47. [CrossRef]

16. Corda, C.; Hendi, S.H.; Katebi, R.; Schmidt, N.O. Effective state, Hawking radiation and quasi-normal modes for Kerr black holes. JHEP 2013, 6, 8. [CrossRef]

17. Jan, K.; Gohar, H. Hawking radiation of scalars from accelerating and rotating black hole with NUT parameter. Astrophys. Space Sci. 2014, 350, 279-284. [CrossRef]

18. Kruglov, S.I. Black hole emission of vector particles in (1+1) dimensions. Int. J. Mod. Phys. A 2014, $29,1450118$. [CrossRef]

19. Matsumoto, M.; Yoshino, H.; Kodama, H. Time evolution of a thin black ring via Hawking radiation. Phys. Rev. D 2014, 89, 044016. [CrossRef]

20. Li, X.Q.; Chen, G.R. Massive vector particles tunneling from Kerr and Kerr-Newman black holes. Phys. Lett. B 2015, 751, 34-38. [CrossRef]

21. Corda, C. Precise model of Hawking radiation from the tunnelling mechanism. Class. Quantum Grav. 2015, 32, 195007. [CrossRef]

22. Anacleto, M.A.; Brito, F.A.; Luna, G.C.; Passos, E.; Spinelly, J. Quantum-corrected finite entropy of noncommutative acoustic balck holes. Annals Phys. 2015, 362, 436. [CrossRef]

23. Lin, H.; Saifullah, K.; Yau, S.T. Accelerating black hole, spin- $\frac{3}{2}$ fields and C-metric. Mod. Phys. Lett. A 2015, 30, 1550044. [CrossRef]

24. Chen, G.R.; Huang, Y.C. Hawking radiation of vector particles as tunneling from the apparent horizon of Vaidya black holes. Int. J. Mod. Phys. A 2015, 30, 1550083. [CrossRef]

25. Anacleto, M.A; Brito, F.A.; Passos, E. Quantum-corrected self-dual black hole entropy in tunneling formalism with GUP. Phys. Lett. B 2015, 749, 181-186. [CrossRef] 
26. Lin, G.; Zu, X. Scalar Particles Tunneling and Effect of Quantum Gravity. J. Appl. Math. Phys. 2015, 3, 134-139.

27. Feng, Z.; Chen, Y.; Zu, X. Hawking radiation of vector particles via tunneling from 4-dimensional and 5-dimensional black holes. Astrophys. Space Sci. 2015, 359, 48. [CrossRef]

28. Saleh, M.; Thomas, B.B.; Kofane, T.C. Hawking radiation from a five-dimensional Lovelock black hole. Front. Phys. 2015, 10, 100401. [CrossRef]

29. Bamba, K.; Odintsov, S.D. Inflationary Cosmology in Modified Gravity Theories. Symmetry 2015, 7, $220-240$. [CrossRef]

30. Övgun, A.; Jusufi, K. Massive Vector Particles Tunneling From Noncommutative Charged Black Holes and its GUP-corrected Thermodynamics. Eur. Phys. J. Plus 2016, 131, 177. [CrossRef]

31. Jusufi, K.; Övgun, A. Hawking Radiation of Scalar and Vector Particles From 5D Myers-Perry Balck Holes. Int. J. Theor. Phys. 2017, 56, 1725. [CrossRef]

32. Cai, Y.F.; Capozziello, S.; Laurentis, M.D.; Saridakis, E.N. f(T) teleparallel gravity and cosmology. Rept. Prog. Phys. 2016, 79, 106901. [CrossRef] [PubMed]

33. Singh, T.I.; Meitei, I.A.; Singh, K.Y. Hawking radiation as tunneling of vector particles from Kerr-Newman black hole. Astrophys. Space Sci. 2016, 361, 103. [CrossRef]

34. Jusufi, K.; Övgun, A. Tunneling of Massive Vector Particles From Rotating Charged Black Strings. Astrophys. Space Sci. 2016, 361, 207. [CrossRef]

35. Li, R.; Zhao, J.K. Massive Vector Particles Tunneling from the Neutral Rotating Anti-di Sitter Black Holes in Conformal Gravity. Commun. Theor. Phys. 2016, 65, 469-472. [CrossRef]

36. Ghrsel, H.; Sakalli, I. Hawking Radiation of Massive Vector Particles From Warped $\mathrm{AdS}_{3}$ Black Hole. Can. J. Phys. 2016, 94, 147-149. [CrossRef]

37. Li, X.Q. Massive vector particles tunneling from black holes influenced by the generalized uncertainty principle. Phys. Lett. B 2016, 763, 80-86. [CrossRef]

38. Nojiri, S.; Odintsov, S.D.; Oikonomou, V.K. Modified Gravity Theories on a Nutshell: Inflation, Bounce and Late-time Evolution. Phys. Rept. 2011, 692, 1-104 . [CrossRef]

39. Corda, C. The future of gravitational theories in the era of the gravitational wave astronomy. Int. J. Mod. Phys. D 2018, 27, 1850060. [CrossRef]

40. Javed, W.; Ali, R.; Abbas, G. Charged Vector Particles Tunneling From Black Ring and 5D Black Hole. Can. J. Phys. 2019, 97, 176-186. [CrossRef]

41. Cvetič, M.; Gubser, S.S. Phases of R-charged Black Holes, Spinning Branes and Strongly Coupled Gauge Theories. JHEP 1999, 9904, 024. [CrossRef]

42. Behrndt, K.; Cvetic, M.; Sabra, W.A. Non-Extreme Black Holes of Five Dimensional N=2 AdS Supergravity. Nucl. Phys. B 1999, 553, 317-332. [CrossRef]

43. Javed, W.; Abbas, G.; Ali, R.; Charged vector particle tunneling from a pair of accelerating and rotating and 5D gauged super-gravity black holes. Eur. Phys. J. C 2017, 77, 296. [CrossRef]

44. Shivalingaswamy, T.; Kagali, B.A. Eigenenergies of a Relativistic Particle in an Infinite Range Linear Potential Using WKB Method. Eur. J. Phys. Educ. 2011, 2, 1309.

(c) 2019 by the authors. Licensee MDPI, Basel, Switzerland. This article is an open access article distributed under the terms and conditions of the Creative Commons Attribution (CC BY) license (http://creativecommons.org/licenses/by/4.0/). 